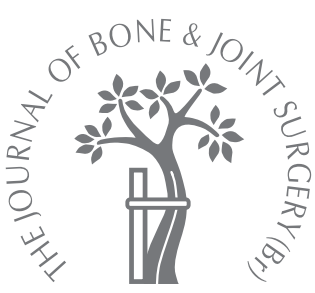

A. P. Rumian, E. R. C. Draper, A. L. Wallace, A. E. Goodship

From the Institute of Orthopaedics and Musculoskeletal Science, University College, London, England

\title{
The influence of the mechanical environment on remodelling of the patellar tendon
}

An understanding of the remodelling of tendon is crucial for the development of scientific methods of treatment and rehabilitation. This study tested the hypothesis that tendon adapts structurally in response to changes in functional loading. A novel model allowed manipulation of the mechanical environment of the patellar tendon in the presence of normal joint movement via the application of an adjustable external fixator mechanism between the patella and the tibia in sheep, while avoiding exposure of the patellar tendon itself. Stress shielding caused a significant reduction in the structural and material properties of stiffness (79\%), ultimate load (69\%), energy absorbed $(61 \%)$, elastic modulus $(76 \%)$ and ultimate stress $(\mathbf{7 2} \%)$ of the tendon compared with controls. Compared with the material properties the structural properties exhibited better recovery after re-stressing with stiffness $97 \%$, ultimate load $92 \%$, energy absorbed $96 \%$, elastic modulus $79 \%$ and ultimate stress $\mathbf{8 0} \%$. The cross-sectional area of the re-stressed tendons was significantly greater than that of stress-shielded tendons.

The remodelling phenomena exhibited in this study are consistent with a putative feedback mechanism under strain control. This study provides a basis from which to explore the interactions of tendon remodelling and mechanical environment.

Disuse of joints may result follow trauma, degenerative change, inflammatory arthropathies, neurological conditions and infection. The treatment of injuries involving bones and joints often involves their immobilisation in the early stages to protect the healing tissue or surgical fixation. However, the actual physiological effects of immobilisation and disuse have only relatively recently been investigated. Joint disuse has a profound effect on the intraand peri-articular tissue, with a sequence of changes including proliferation of intraarticular connective tissue, the formation of adhesions and ulceration of the cartilage, with joints becoming stiff as a result of muscular and capsular contracture combined with intraarticular ankylosis. ${ }^{1,2}$

Biomechanical properties of tendons and ligaments can be divided into structural, such as the cross-sectional area, the length, the ultimate load and stiffness, which relate to the function of the structure as a whole, and material including stress, strain and elastic modulus which relate to the attributes of the constituent tissue. Noyes et $\mathrm{al}^{3}$ and Noyes ${ }^{4}$ investigated the effect of immobilisation and reconditioning on the femur-anterior cruciate ligament (ACL)tibia complex in primates. After eight weeks of immobilisation of the limb in a plaster cast, the bone-ACL complex had an ultimate load of $61 \%$ and energy absorbed of $68 \%$ compared with controls, with a significant reduction in stiffness to $69 \%$. This finding of reduced stiffness in the ligament was in contrast to previous assumptions that immobility causes contracture and stiffness in all the peri-articular tissues. ${ }^{5-7}$ In animals that were allowed normal movement for five months after eight weeks of immobilisation, the ultimate load and energy absorbed were still only $76 \%$ and $78 \%$, respectively, compared with the control values, whereas after 12 months of reconditioning they were $91 \%$ and $92 \%$, respectively, demonstrating that a short period of disuse can result in long-term consequences.

Amiel et $\mathrm{al}^{8}$ tested the femur-lateral collateral ligament (LCL)-tibia complex from rabbit knees immobilised for nine weeks. The load-elongation curve was similar to that reported by Noyes et al. ${ }^{3}$ By demonstrating that the cross-sectional area had not decreased significantly, whereas the stressstrain curve showed significantly inferior mechanical properties, Amiel et $\mathrm{al}^{8}$ deduced that the changes must be due to alterations in the substance of the ligament itself, rather 
than simple atrophy of the tissue. Analysis of the patellar tendon and medial collateral ligament (MCL) from the immobilised knees showed an increased rate of both collagen synthesis and degradation, with no change in the total collagen mass. He suggested that newly synthesised collagen was laid down in a haphazard manner owing to the lack of the normal mechanical stimulus, resulting in inferior mechanical properties.

The development of more sophisticated methods of measuring cross-sectional area and the use of video technology allowed for more accurate measurement of biomechanical properties, enabling the study of the stressstrain characteristics of tendon and ligament tissue itself. Woo et $\mathrm{al}^{9}$ found a significant reduction in the crosssectional area of MCLs from rabbit knees immobilised for nine and 12 weeks. The cross-sectional area of MCLs from knees remobilised for nine weeks after nine weeks of immobilisation was not significantly different from that of the controls. Immobilisation was again shown to significantly reduce both the structural and the material properties, with a reduction in ultimate load and tensile strength to $31 \%$ and approximately $54 \%$ respectively, compared with the controls. After remobilisation, the stress-strain behaviour of the experimented MCLs was not different from controls, but the ultimate loads were still lower, i.e., the material properties of the MCL appeared to recover faster than the structural properties. Larsen, Forwood and Parker ${ }^{10}$ compared the effects of immobilisation on the $\mathrm{ACL}$ and the posterior cruciate ligament (PCL) from rat knees immobilised in a plaster cast for four weeks. The ultimate load of the ACL was significantly reduced, whereas that of the PCL was not, indicating that different connective tissue structures from the same joint react specifically to alterations in loading.

The dynamics of an immobilisation model may result in very different forces being experienced by the periarticular structures, resulting in variations in their response. The tendons will tend to experience a continuous load owing to the resting tone of their muscles. The actual amount of stress deprivation induced in individual tissues by immobilisation has not been quantified, and the changes observed in the peri-articular tissues could be due to the position of immobilisation, a change in weightbearing or other causative factors produced by joint disuse. The evidence from previous studies is contradictory. For example, nine weeks of immobilisation have been reported to cause a $50 \%$ reduction in elastic modulus, with no change in cross-sectional area, ${ }^{8}$ no change in elastic modulus with a reduction in cross-sectional area to $74 \%,{ }^{11}$ or a reduction in both elastic modulus and crosssectional area. ${ }^{9}$ Thus, although the studies discussed above show that changes do occur in ligaments and tendons after immobilisation, the precise nature of the causative factor, whether it be decreased strain, decreased stress, an inflammatory response, or some other unknown mediator, cannot be specified.
Yamamoto et $\mathrm{al}^{12}$ studied the direct effect of stress shielding the patellar tendon in the rabbit while preserving joint movement. The patellar tendon was exposed surgically and a cerclage wire passed between the tendon and the tibia. The wire was tightened to slacken the tendon and the wound closed. They found a reduction in the elastic modulus and ultimate stress of the tendon, together with a reduction in tendon length and an increase in cross-sectional area. The biomechanical effects depended on the degree of stress reduction. ${ }^{13}$ Few data exist on the effect of restressing a tendon that has been stress shielded in the presence of joint movement: Yamamoto et $\mathrm{al}^{14}$ showed that restressing the patellar tendon of the rabbit with normal activity after two weeks of stress-shielding produced a recovery of the properties of the tendon, but they were still significantly worse than controls even after 12 weeks of restressing. These studies have an advantage over those using an immobilisation model, in that an individual ligament or tendon was isolated while attempting to recreate a normal mechanical environment for the rest of the joint and the peri-articular tissues.

The remodelling of tendons and ligaments is of great interest owing to the increased use of surgical techniques such as ACL reconstruction using both auto- and allograft tissue, ${ }^{15}$ or rotator cuff repair. In rotator cuff tears it has recently been demonstrated that part of the observed tissue retraction is due to remodelling at the myotendinous junction, and not just due to muscle shortening. ${ }^{16}$ In reconstruction of the ACL with autograft tendon, a process of 'ligamentisation' has been described, with gradual metaplasia occurring over several years. ${ }^{17}$ It has been suggested that the properties of the fibroblasts that populate tendon grafts differ from those found in normal tendons, resulting in a lower potential for remodelling. ${ }^{18,19}$ Both excessive and insufficient stress can be detrimental. Tohyama and Yasuda ${ }^{20}$ noted a reduction in the mechanical properties of a tendon autograft model as a result of increasing the applied stress.

There has been considerable interest in the potential to manipulate the remodelling process using agents such as growth factors and cytokines. A recent experimental study has investigated the use of osteogenic protein- 1 to stimulate ligament remodelling in a rabbit model of ACL injury. ${ }^{21}$ However, although mechanical stimuli have been shown to have a significant long-lasting effect on tendons and ligaments, as discussed above, the main controlling stimulus for remodelling has yet to be clarified. Thus, most rehabilitation programmes following injury or surgery have an empirical rather than a quantified scientific basis. An understanding of tissue remodelling in response to functional demand would provide a scientific basis for training and rehabilitation regimens that could maximise benefit and minimise the risk of injury.

The patellar tendon is of particular interest as it serves as a suitable model for 'general' tendon behaviour ${ }^{22}$ and is frequently encountered in clinical practice, either as a graft for 


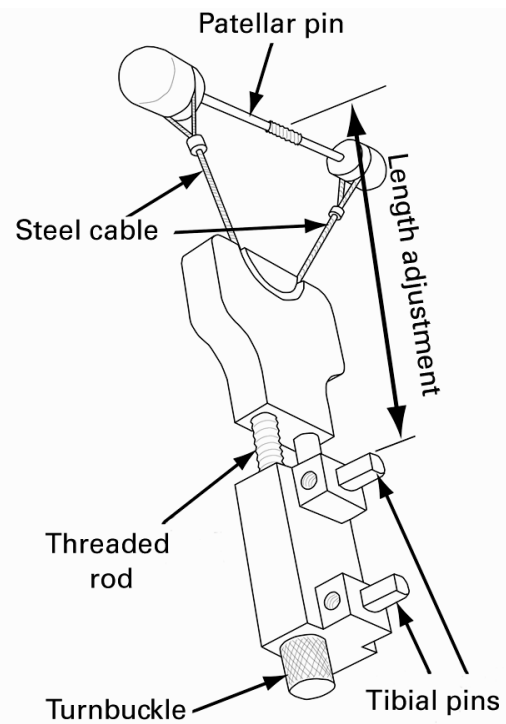

Fig. 1a

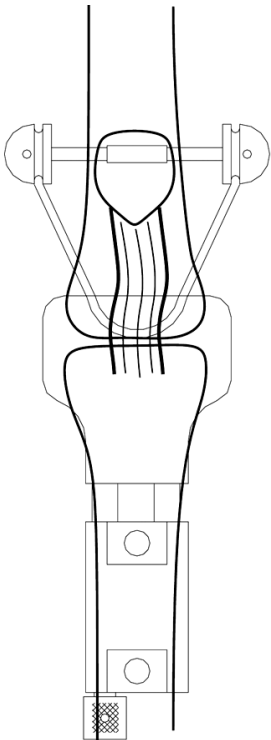

Fig. 1b

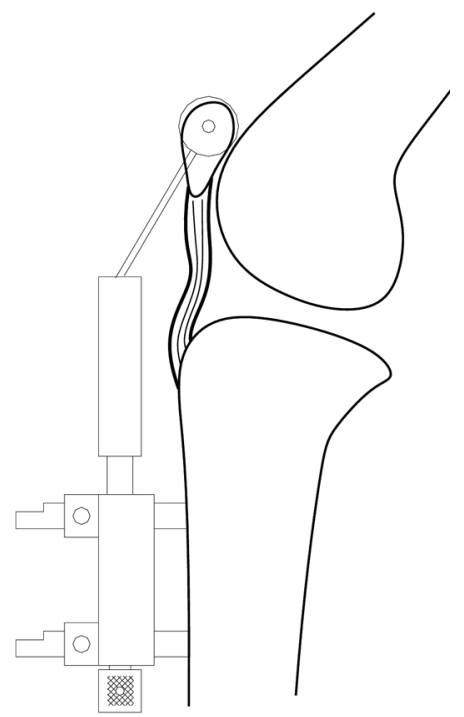

Fig. 1c

Stress shielding the patellar tendon. Diagram showing a) the custom-built external fixator, consisting of a tibial component, a patellar component and a cable linkage. b) and c) show the fixator as applied to the animal in the coronal and sagittal plane, respectively. The tibial component was fixed to the tibia using two $5 \mathrm{~mm}$ diameter bone half-pins (Orthofix Inc., McKinney, Texas) inserted percutaneously. A $3 \mathrm{~mm}$ pin was drilled transversely through the body of the patella. An end cap on each side accepted a wire linkage to the tibial block. After the external fixator was applied, the distance of the inferior pole of the patella from the tibial tuberosity was measured with a pair of Vernier calipers with the knee in $90^{\circ}$ of flexion. The patella was then drawn 15 mm towards the tibia by using the turnbuckle screw mechanism. This was confirmed by a repeated measurement. The range of movement of the knee was examined and found not to be limited by this procedure. c) The patellar tendon was palpated to be slack in full flexion, $90^{\circ}$ of flexion and full extension. This was assumed to reflect complete stress shielding of the tendon. During the period of stress shielding, the tendon was palpated in $90^{\circ}$ of knee flexion twice a week to confirm that it was still slack.

use in ACL reconstruction, or involved in disease or injury itself. The aim of this study using a novel animal model was to test the hypothesis that tendon exhibits structural adaptation in response to functional loading. The model was used to investigate the effects of stress shielding on the patellar tendon in sheep, and how these effects might be reversed by reconditioning after stress shielding. The model uses a technique that avoids direct exposure or contact with the patellar tendon itself.

\section{Materials and Methods}

The patellar tendon in the sheep has been shown to be a suitable structure for studying tendon biology. ${ }^{22}$ It was chosen for use in this study because the distribution of force and tissue quality have been shown to be fairly uniform throughout the tendon. ${ }^{23}$ Surgically, it is easily accessible and the bony attachments to the patella and tibia allow the secure insertion of metalwork, and its functional environment can be manipulated while allowing full joint function.

A change in ultimate stress of $30 \%$ was deemed to be clinically important, and a power analysis suggested that a minimum of four subjects and controls would be required for an unpaired $t$-test to reach statistical significance, assuming an SD of $10 \%$ (power $0.8, \alpha 0.05$ ). Two groups of animals were used. In group 1 , six sheep had the right patellar tendon stress shielded for six weeks before being killed. In group 2, six sheep had the right patellar tendon stress shielded for six weeks, after which the stress shielding device was removed, allowing physiological re-stressing for a further six weeks. Biomechanical analysis was performed on the treated tendons and the untreated contralateral tendons were similarly analysed to obtain control data for comparison.

Animals. There were 12 skeletally mature Welsh Mule ewes who were housed extensively in large indoor pens. All the animal procedures were carried out under licences granted by the United Kingdom Home Office in accordance with the Animals (Scientific Procedures) Act of 1986, and had local ethical committee approval. The animals were killed by lethal intravenous injection of barbiturate.

Stress shielding the patellar tendon. A custom-built external fixator was used to stress shield the patellar tendon in the presence of normal joint movement, as shown in Figure 1.

The surgical procedures were performed under general anaesthesia using a sterile technique. Pre-medication with $0.05 \mathrm{ml} / \mathrm{kg} 2 \%$ xylazine was administered intramuscularly. Induction was achieved with $0.2 \mathrm{ml} / \mathrm{kg}$ ketamine and $2.5 \mathrm{mg}$ midazolam intravenously. Buprenorphine was used for analgesia. The sheep were then intubated. A oesophageal tube was used to void rumenal gases, and anaesthesia was maintained with $2 \%$ halothane gas.

Analysis of structural and material properties. The patellar tendon from each limb was harvested as a bone-patellar tendon-bone unit and immediately subjected to testing. The samples were moistened throughout with physiological saline. $^{24}$ 


\begin{tabular}{|c|c|c|c|}
\hline & Control & Stress-shielded & Re-stressed \\
\hline Length (mm) & $38.7(1.04)$ & $35.4(2.16)$ & $36.0(0.95)$ \\
\hline $\operatorname{CSA}^{*}\left(\mathrm{~mm}^{2}\right)$ & $38.3(1.43)$ & $36.9(1.77)$ & $43.5(1.20)$ \\
\hline Stiffness (kN/mm) & $0.35(0.013)$ & $0.28(0.025)$ & $0.34(0.029)$ \\
\hline Ultimate force (kN) & $2.92(0.075)$ & $2.02(0.237)$ & $2.68(0.282)$ \\
\hline Energy absorbed (MPa) & $16.5(1.07)$ & $10.0(2.20)$ & $15.9(2.51)$ \\
\hline
\end{tabular}

* CSA, cross-sectional area

Measurement of the cross-sectional area. This was measured using the method described by Goodship and Birch. ${ }^{25}$ An alginate dental impression material (Blueprint cremix, Dentsply De Trey, Germany; supplied by Claudius Ash, Potters Bar, United Kingdom) was used to make an impression of the bone-patellar tendon-bone complex. After removal of the specimen, transverse sections were taken through the midpoint of the tendon mould, and at points $10 \mathrm{~mm}$ either side. The sections were photographed on a calibration scale using a mounted 5 megapixel digital camera. Digital images were used to measure the area of the cavity left by the specimen in the mould sections using a semi-automated image analysis software package (Image-Pro Plus, Media Cybernetics Inc., Bethesda, Maryland). The mean of three measurements was calculated to obtain the value for the cross-sectional area of the tendon.

Tendon length. The tendon length was defined as the distance from the most distal part of its insertion into the patella to the most proximal part of its insertion into the tibia. This was measured with a Vernier caliper.

Mechanical testing. The bone-tendon unit was mounted in the specifically designed clamps of a computer-controlled servohydraulic materials testing machine (Dartec/Zwick, GmbH \& Co., Ulm, Germany), which combined pin fixation with a cryo-clamp technique. ${ }^{26}$ Thus, the gauge length of the specimen being tested was the distance between the two clamps.

The tendon was pre-conditioned to obtain a steady-state load-deformation curve ${ }^{27,28}$ using a standard protocol of 20 cycles of loading from $5 \mathrm{~N}$ to $50 \mathrm{~N}$ at $0.5 \mathrm{~Hz} .^{29}$ It was then tested to failure under displacement control at a distraction rate of $40 \mathrm{~mm} / \mathrm{s}$, which is comparable to a rate of loading reported in the patellar tendon of the goat under physiological conditions. ${ }^{30}$ Stiffness was defined as the gradient of the linear part of the load-deformation curve. Likewise, elastic modulus was the gradient of the stress-strain curve. The ultimate load, ultimate stress, and ultimate strain were derived from peak values before failure. Strain energy was defined as the area under the stress-strain curve. Toe limit strain was defined as the maximum strain of the initial portion of the stress-strain curve before the onset of linear elastic behaviour. The mode of failure of each tendon was also recorded.

Statistical method. All the results are reported as the mean \pm the standard error of the mean (SEM). The Shapiro-Wilks test was used to confirm the normality of distribution of the data.
Data from the untreated tendons of both groups were tested for statistical significance using the unpaired $t$-test. A one-way analysis of variance was used to examine data between the two experimental groups and controls. Where statistical differences were found, these were further explored using the Tukey-Kramer method for multiple comparisons. The level of statistical significance was set at $\mathrm{p}<0.05$.

\section{Results}

In group 1, one animal was excluded because of an infection of the pin site in the patella. In group 2, one sheep died immediately after anaesthesia. This left five animals in each group. The Shapiro-Wilks test revealed no evidence of non-normality of distribution of the data. There were no significant differences between groups 1 and 2 in any of the parameters of the untreated tendons. Therefore, these results were pooled and used as the control data.

Mode of failure. All the tendons failed in the substance of the ligament. Of the ten control tendons, seven failed in the middle, two in the proximal and one in the distal third. All the stress-shielded tendons in group 1 failed in the middle third. Out of the five re-stressed tendons, four failed in the middle and one in the proximal third. None failed due to bone avulsion. No slippage was observed to have occurred in the testing clamps.

Structural properties. The tendon lengths did not differ significantly between the three groups. However, the cross-sectional area of the re-stressed tendons was found to be significantly greater than the stress-shielded tendons, with a mean difference of $6.6 \mathrm{~mm}^{2}$ ( $\mathrm{p}=0.046$; 95\% confidence interval (CI) 0.1 to 13.2). Neither the stressshielded nor the re-stressed tendons were significantly different from the controls.

The stress-shielded tendons exhibited statistically significant reduction compared with the controls, with a mean stiffness of $79 \%$ (mean difference $0.074 \mathrm{kN} / \mathrm{mm} ; \mathrm{p}=0.041$; $95 \%$ CI 0.003 to 0.14 ), an ultimate load of $69 \%$ (mean difference $0.90 \mathrm{kN} ; \mathrm{p}=0.0042 ; 95 \%$ CI 0.29 to 1.5$)$ and energy absorbed of $61 \%$ (mean difference $6.5 \mathrm{MPa}$; $\mathrm{p}=0.039 ; 95 \%$ CI 0.32 to 13$)$. However, the structural properties of the re-stressed tendons were much closer in value to, and not significantly different from, the control tendons, with a mean stiffness of $97 \%$, an ultimate load of $92 \%$ and energy absorbed of $96 \%$. The values for 


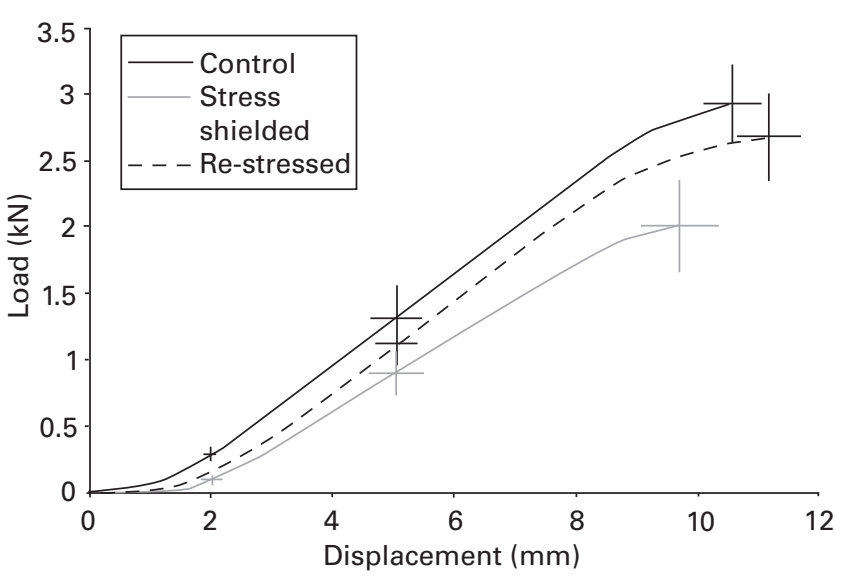

Fig. 2a

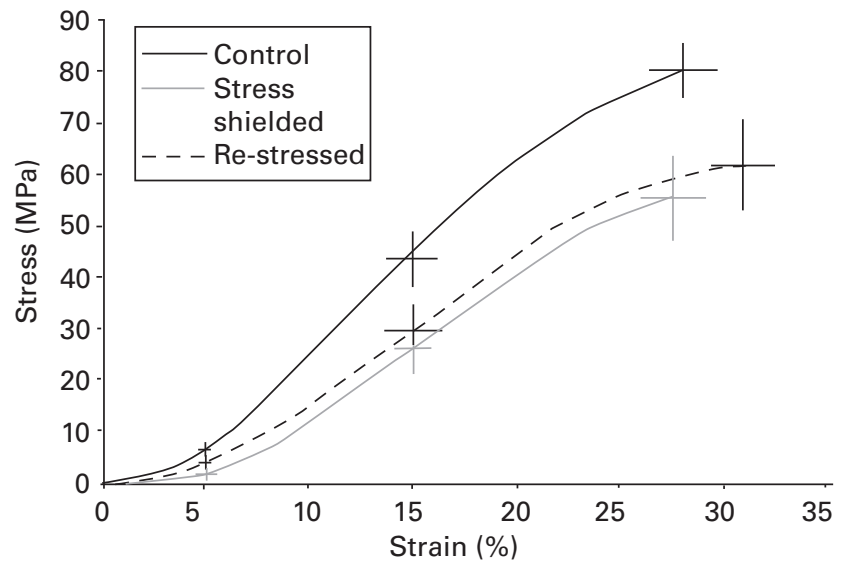

Fig. $2 b$

Graphs showing a) structural properties: load-deformation curve for control, stress shielded and re-stressed tendons, the gradient of the linear portion of the curve represents stiffness, error bars show standard error of the mean and b) material properties: stress-strain curve, the gradient of the linear portion represents the elastic modulus.

Table II. Material properties of control, stress shielded and re-stressed tendons reported as mean values, with standard error in parentheses

\begin{tabular}{lclclcl}
\hline & \multicolumn{2}{c}{ Control } & \multicolumn{2}{c}{ Stress-shielded } & Re-stressed \\
\hline Ultimate stress (MPa) & 76.9 & $(2.66)$ & 55.3 & $(6.69)$ & 61.9 & $(6.95)$ \\
Ultimate strain (\%) & 28.1 & $(0.80)$ & $27.5(1.4)$ & $31.0(1.4)$ \\
Elastic modulus (MPa) & $373 \quad(16.7)$ & 284 & $(25.1)$ & $296 \quad(28.0)$ \\
Toe limit strain (\%) & $3.55(0.5)$ & 7.2 & $(2.4)$ & $5.0(0.4)$ \\
\hline
\end{tabular}

structural properties are shown in Table I. The loaddeformation curves are shown in Figure $2 \mathrm{a}$.

Material properties. The stress-shielded tendons also exhibited significant deterioration in material properties compared the controls, with a mean elastic modulus that was $76 \%$ of the control value (mean difference $88 \mathrm{MPa}$; $\mathrm{p}=0.026 ; 95 \%$ CI 10 to 167$)$ and a mean ultimate stress of $72 \%$ (mean difference $22 ; \mathrm{p}=0.012 ; 95 \%$ CI 4.6 to 39 ). After re-stressing these values only recovered to $79 \%$ and $80 \%$, respectively, although this was not found to be significantly different from either the control or the stressshielded tendons. The ultimate strain was similar for all tendons, as was the toe limit strain. The values for material properties are shown in Table II. Figure 2 b shows the stressstrain curves for all experimental groups.

\section{Discussion}

In this study the patellar tendon exhibited significant changes in its biomechanical characteristics, both structural and material, in response to changes in functional loading. All the stress-shielded tendons failed in the mid-substance, indicating that change occurred in the tendon itself rather than at the sites of bony insertion. If the sites of insertion were more affected or there was significant resorption of bone, then one would expect more failures at these sites or by bone avulsion, as described by Woo et $\mathrm{al}^{9}$ and Noyes ${ }^{4}$ in immobilised joints. Fujie et $\mathrm{al}^{31}$ also observed that stress shielding of the patellar tendon for more than one week in both mature and immature rabbits resulted in failures in the midsubstance of the tendon.

Stress shielding for six weeks did not significantly change the tendon length or the cross-sectional area. This differs from the findings of Yamamoto et al, ${ }^{12}$ who observed an increased cross-sectional area of $140 \%$ compared with controls and a shorter tendon length after six weeks of stress shielding. However, they noted that the cross-sectional area decreased between three and six weeks of stress shielding, which they could not explain. Also, in the canine ACL, the cross-sectional area has been observed to increase after six weeks of stress shielding, but this effect had disappeared by 12 weeks. ${ }^{32}$ Changes in the cross-sectional area could be related to changes in water and glycosaminoglycan content, or the collagen mass. ${ }^{33,34}$ The lack of difference in toe limit strain supports the observation of no change in tendon length. This limit is dependent on the crimp profile of the tendon, which would not be expected to change in the absence of change in length.

Re-stressing tendons after two or three weeks of stressshielding has been reported to reduce the cross-sectional area and increase the tendon length. ${ }^{14}$ However, we found 


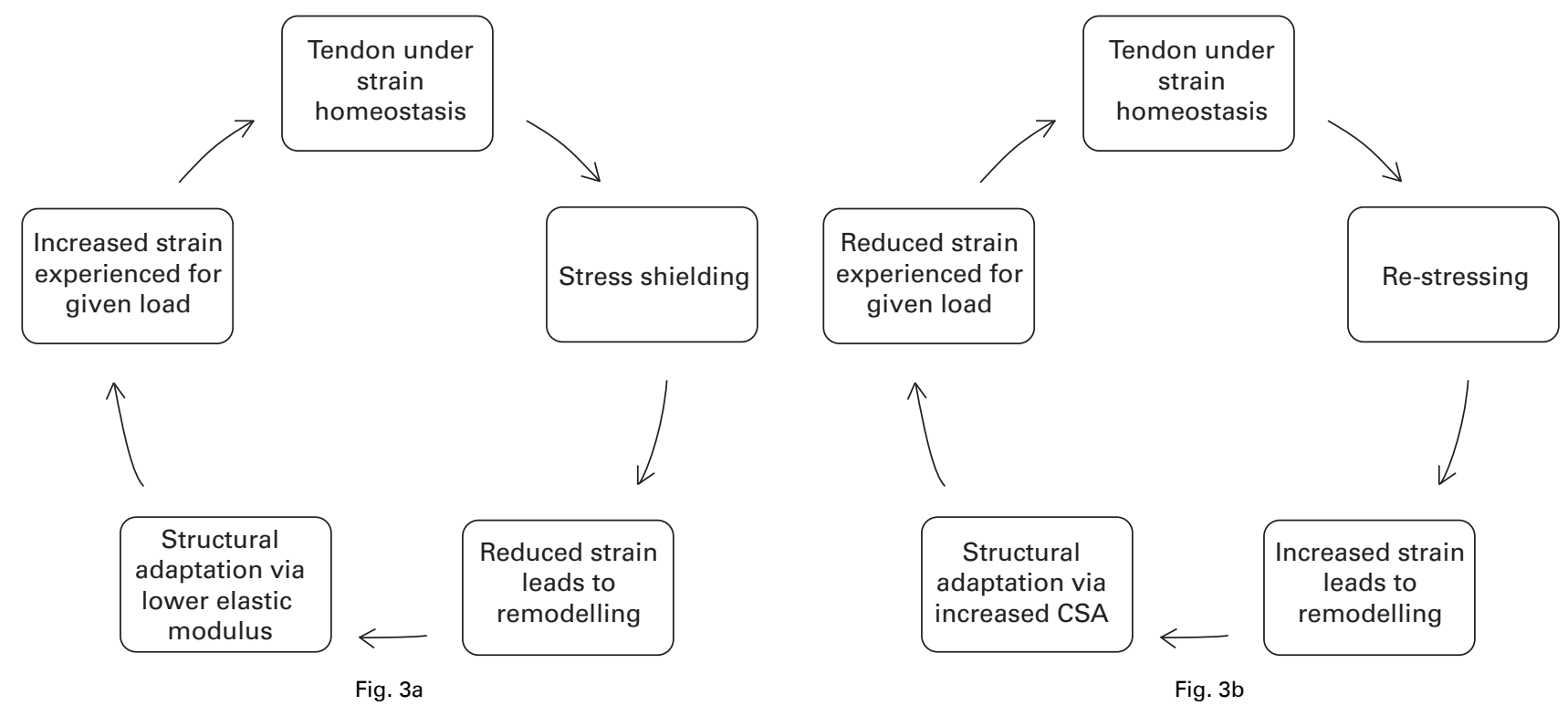

Proposed 'strain homeostasis' feedback control loops governing remodelling behaviour for a) stress shielded and b) re-stressed tendons (CSA, crosssectional area).

that the cross-sectional area was significantly increased in re-stressed tendons compared with the stress-shielded tendons, although these findings are not directly comparable, owing to the different lengths of initial stress shielding.

Both the structural and the material properties of the patellar tendon were significantly reduced by stress shielding. The stiffness and elastic modulus were $79 \%$ and $76 \%$ of the control values, respectively, whereas the ultimate load and ultimate stress were $69 \%$ of the controls. Yamamoto et $\mathrm{al}^{12}$ found much greater reductions in the stressshielded patellar tendon in the rabbit knee, with a minimum modulus of approximately $20 \%$ of controls after six weeks of stress-shielding. This is in contrast to Keira et al, ${ }^{32}$ who observed no significant change in modulus after six weeks of stress-shielding of the canine ACL, but a modulus of $61 \%$ of the control value after 12 weeks. The patellar tendon of the sheep appears to lie in the middle of this spectrum.

Re-stressing tendons for six weeks after six weeks of stress shielding demonstrated a recovery of structural properties, with a return to almost $100 \%$ of the control values. This was associated with an increase in cross-sectional area. However, the material properties exhibited a trend towards incomplete recovery, achieving only $80 \%$ of the control values. Thus, the tendons are shown to experience structural adaptation. The re-stressed tendons compensate for their continued low elastic modulus by the increase in the cross-sectional area.

A possible reason for the disagreement between studies regarding the effect of stress shielding on the biomechanical properties of tendons and ligaments could be differences in the patterns of activity of the experimental animals, which have not been accurately quantified in any of the studies, or due to differing metabolic responses of the tissues. ${ }^{34}$ Another explanation may be the different strain rates used in various studies. Those using video analysis systems have employed low strain rates to enable the capture of images, whereas the physiological strain rate used in our study is much higher. Thus the differences in findings could be due to the sensitivity of tendon to strain rate. Noyes, DeLucas and Torvik $^{35}$ found that, for a 100 -fold increase in test speed, the failure load increased by $21 \%$.

Another possible reason is the disparate nature of the techniques used to achieve the stress shielding. Immobilisation models are unable to achieve stress shielding of an individual structure without affecting the other tissues around a joint. Previous techniques to functionally isolate one structure have involved quite extensive surgical exposure and dissection of the structure under investigation. This, of necessity, induces an inflammatory healing response directly adjacent to the structure, which may then influence its response to the altered mechanical environment. For example, in the study by Keira et $\mathrm{al}^{32}$ on the canine ACL, sham operated controls still varied significantly from nonoperated controls. In Yamamoto's model, sham operation did not cause significant differences in the patellar tendon compared with controls. However, this does not exclude the possibility that the inflammatory response still modulated the behaviour of the tendon to stress shielding. In order to control for this it would require comparison of the results of stress shielding the tendon by two different techniques, one using the surgical exposure and one avoiding it. The novel model presented here involves the use of bone pins inserted into a site remote from the patellar tendon itself, and the stress shielding is achieved without the need for any surgical insult to the structure under investigation. 
There are several weaknesses in this study, a major one of which is the small number of animals. An initial power analysis suggested that only four animals would be required to show a $30 \%$ reduction in ultimate stress to be statistically significant. The initial study design included six animals in each group, although only five remained for analysis. Despite this, statistically significant differences were found between control and stress shielded tendons. The re-stressed tendons, however, exhibited better biomechanical properties than suggested by previous reports, and the SD was larger than the $10 \%$ initially assumed. Thus, the study lacked the power to detect differences between the restressed and the other tendons. Another weakness is the lack of histological or molecular data to correlate with the results of mechanical testing. It was felt wrong to perform such analysis on the same tissue, as the results might be influenced by the disruption of extracellular matrix and intracellular architecture involved in destructive mechanical testing.

The biomechanical tests were carried out using a cryoclamp technique with supplementary pin fixation. The specimens were mounted carefully so that the length of the tendon would be equal to the length of gauge submitted to testing. Although no slippage was observed to have occurred in the clamps during testing, this was not directly measured. Also, changes in the sites of insertion of the patellar tendon may have contributed to the observed effects of the stress shielding. However, the fact that all tendons failed in the mid-substance supports the assumption that most change occurred in the tendon itself, and that slippage was not occurring in the clamps. Use of a video analysis system to determine strain of the tendon midsubstance directly would have avoided this uncertainty. However, the limitations of such a system would not have allowed mechanical testing at the high, physiologically based strain rate used in this study.

Although the biomechanical changes in the tendon have been described here, it is difficult to ascertain how much deterioration needs to occur before becoming clinically relevant, or how much recovery is required before full normal activity can be tolerated. The initial aim in this study was to detect a $30 \%$ reduction in ultimate stress, as this would be the level at which a catastrophic failure of the tendon might occur in vivo. However, it may be that the elastic modulus itself is of more significance in allowing normal function during the stresses experienced in routine physiological activity.

The nature of the changes in the tissue of a stress-shielded tendon is just beginning to be clarified. These include an increase in the number of fibroblasts, ${ }^{12,36}$ of the number of small collagen fibrils, ${ }^{36,37}$ the expression of manganese superoxide dismutase, ${ }^{38}$ and the expression of cytokines such as IL-1 $\beta$, TNF- $\alpha$ and TGF- $\beta .{ }^{39}$ Abiezzi et al ${ }^{40}$ described an increase in integrin adhesion subreceptor units in conjunction with the remodelling of stress-deprived rabbit ligaments. Mechanical strain has recently been shown to stimulate the conformational activation of integrins directly. ${ }^{41}$

The biomechanical changes observed in the present study could be controlled by a feedback loop under strain control (Fig. 3), similar to that described in a recent computational model. ${ }^{42}$ When deprived of stress, the strains experienced by a tendon are much lower than normal. Mechanotransduction mechanisms, possibly mediated by integrins, initiate the remodelling process. As the elastic modulus decreases, the strain increases until 'strain homeostasis' is achieved. When once again subjected to stress, the weakened tendons experience greater strains than normal. Mechanotransduction mechanisms result in structural adaptation, with an increase in the cross-sectional area. This once more brings the strain back towards normal values. Although the tissue exhibits structural adaptation, the evidence from this and other studies suggests that changes in material properties due to stress deprivation are not quickly reversed.

Much further work using this model is planned. Initially, the histological and biochemical changes occurring in the stress shielded and re-stressed tendons need to be elucidated. Subsequently, the effects of increased stress on normal tendons can be investigated by applying a distracting force across the components of the external fixator mechanism. Mechanical actuators will be used so that the strain applied can be accurately controlled in terms of rate, cyclical frequency and the number of cycles. One of the primary aims for the future will be to determine the threshold levels of stimulation required to control the remodelling process. This would be of direct clinical relevance and enable us to elucidate the least amount of activity necessary to prevent deterioration, when any extra activity ceases to be of benefit; and how much is too much.

No benefits in any form have been received or will be received from a commercial party related directly or indirectly to the subject of this article.

\section{References}

1. Evans EB, Eggers GW, Butler JK, Blumel J. Experimental immobilization and remobilization of rat knee joints. J Bone Joint Surg [Am] 1960;42-A:737-58.

2. Enneking WF, Horowitz M. The intra-articular effects of immobilization on the human knee. J Bone Joint Surg [Am] 1972;54-A:973-85.

3. Noyes FR, Torvik PJ, Hyde WB, DeLucas JL. Biomechanics of ligament failure. II: an analysis of immobilization, exercise, and reconditioning effects in primates. J Bone Joint Surg [Am] 1974;56-A:1406-18.

4. Noyes FR. Functional properties of knee ligaments and alterations induced by immobilization: a correlative biomechanical and histological study in primates. Clin Orthop 1977;123:210-42.

5. Akeson WH. An experimental study of joint stiffness. Am J Orthop 1961;43A:1022-34

6. Akeson WH, Amiel D, LaViolette D. The connective-tissue response to immobility: a study of the chondroitin-4 and 6-sulfate and dermatan sulfate changes in periarticular connective tissue of control and immobilized knees of dogs. Clin Orthop 1967; 51:183-97.

7. Akeson WH, Amiel D, LaViolette D, Secrist D. The connective tissue response to immobility: an accelerated ageing response? Exp Gerontol 1968;3:289-301.

8. Amiel D, Woo SL, Harwood FL, Akeson WH. The effect of immobilization on collagen turnover in connective tissue: a biochemical-biomechanical correlation. Acta Orthop Scand 1982;53:325-32. 
9. Woo SL, Gomez MA, Sites TJ, et al. The biochemical and morphological changes in the medial collateral ligament of the rabbit after immobilization and remobilization. J Bone Joint Surg [Am] 1987;69-A:1200-11.

10. Larsen NP, Forwood MR, Parker AW. Immobilization and retraining of cruciate ligaments in the rat. Acta Orthop Scand 1987;58:260-4.

11. Newton PO, Woo SL, MacKenna DA, Akeson WH. Immobilization of the knee joint alters the mechanical and ultrastructural properties of the rabbit anterior cruciate ligament. J Orthop Res 1995;13:191-200.

12. Yamamoto N, Ohno K, Hayashi K, et al. Effects of stress shielding on the mechanical properties of rabbit patellar tendon. J Biomech Eng 1993;115:23-8.

13. Majima T, Yasuda K, Fujii T, et al. Biomechanical effects of stress shielding of the rabbit patellar tendon depend on the degree of stress reduction. J Orthop Res 1996;14:377-83

14. Yamamoto N, Hayashi K, Kuriyama $\mathbf{H}$, et al. Effects of restressing on the mechanical properties of stress-shielded patellar tendons in rabbits. J Biomech Eng 1996;118:216-20.

15. Robertson A, Nutton RW, Keating JF. Current trends in the use of tendon allografts in orthopaedic surgery. J Bone Joint Surg [Br] 2006;88-B:988-92.

16. Meyer DC, Lajtai G, von Rechenberg B, Pfirmann CWA, Gerber C. Tendon retracts more than muscle in experimental chronic tears of the rotator cuff. $J$ Bone Joint Surg [Br] 2006;88-B:1533-8.

17. Deehan DJ, Cawston TE. The biology of integration of the anterior cruciate ligament. J Bone Joint Surg [Br] 2005;87-B:889-95.

18. Ikema Y, Tohyama H, Nakamura H, Kanaya F, Yasuda K. Growth kinetics and integrin expression of fibroblasts infiltrating devitalised patellar tendons are different from those of intrinsic fibroblasts. J Bone Joint Surg [Br] 2005;87-B:1689-93

19. Tohyama H, Yasuda K, Uchida H, Nishihara J. The responses of extrinsic fibroblasts infiltrating the devitalised patellar tendon to IL-1 [beta] are different from those of normal tendon fibroblasts. J Bone Joint Surg [Br] 2007;89-B:1261-7.

20. Tohyama $\mathbf{H}$, Yasuda $\mathbf{K}$. The effect of increased stress on the patellar tendon. J Bone Joint Surg [Br] 2002;84-B:440-6.

21. Hayashi R, Kondo E, Tohyama H, Saito T, Yasuda K. In vivo local administration of osteogenic protein-1 increases structural properties of the overstretched anterior cruciate ligament with partial midsubstance laceration: a biomechanical study in rabbits. J Bone Joint Surg [Br] 2008;90-B:1392-400

22. Rumian AP, Wallace AL, Birch HL. Tendons and ligaments are anatomically distinct but overlap in molecular and morphological features: a comparative study in an ovine model. J Orthop Res 2007;25:458-64.

23. Yamamoto N, Hayashi K, Kuriyama H, et al. Mechanical properties of the rabbit patellar tendon. J Biomech Eng 1992;114:332-7.

24. Woo SL, Gomez MA, Seguchi Y, Endo CM, Akeson WH. Measurement of mechanical properties of ligament substance from a bone-ligament-bone preparation. J Orthop Res 1983;1:22-9.

25. Goodship AE, Birch HL. Cross sectional area measurement of tendon and ligament in vitro: a simple, rapid, non-destructive technique. J Biomech 2005;38:605-8.
26. Riemersa DJ, Schamhardt HC. The cryo-jaw, a clamp designed for in vitro rheology studies of horse digital flexor tendons. J Biomech 1982;15:619-20.

27. Schatzmann L, Brunner P, Stäubli HU. Effect of cylic preconditioning on the tensile properties of human quadriceps tendons and patellar ligaments. Knee Surg Sports Traumatol Arthrosc 1998;6(Suppl):56-61.

28. Yahia LH, Drouin G. Study of the hysteresis phenomenon in canine anterior cruciate ligaments. J Biomed Eng 1990;12:57-62.

29. Batson EL, Paramour RJ, Smith TJ, et al. Are the material properties and matrix composition of equine flexor and extensor tendons determined by their functions? Equine Vet J 2003;35:314-18

30. Korvick DL, Cummings JF, Grood ES, et al. The use of an implantable force transducer to measure patellar tendon forces in goats. J Biomech 1996;29:557-61.

31. Fujie H, Yamamoto N, Murakami T, Hayashi K. Effects of growth on the response of the rabbit patellar tendon to stress shielding: a biomechanical study. Clin Biomech (Bristol, Avon/2000;15:370-8.

32. Keira M, Yasuda K, Kaneda K, Yamamoto N, Hayashi K. Mechanical properties of the anterior cruciate ligament chronically relaxed by elevation of the tibial insertion. J Orthop Res 1996;14:157-66.

33. Akeson WH, Amiel D, Abel MF, Garfin SR, Woo SL. Effects of immobilization on joints. Clin Orthop 1987;219:28-37.

34. Harwood FL, Amiel D. Differential metabolic responses of periarticular ligaments and tendon to joint immobilization. J Appl Physiol 1992;72:1687-91.

35. Noyes FR, DeLucas JL, Torvik PJ. Biomechanics of anterior cruciate ligament failure: an analysis of strain-rate sensitivity and mechanisms of failure in primates. $J$ Bone Joint Surg [Am] 1974;56-A:236-53.

36. Muellner T, Kwasny 0, Loehnert V, et al. Light and electron microscopic study of stress-shielding effects on rat patellar tendon. Arch Orthop Trauma Surg 2001;121:561-5.

37. Majima T, Yasuda K, Tsuchida T, et al. Stress shielding of patellar tendon: effect on small-diameter collagen fibrils in a rabbit model. J Orthop Sci 2003;8:836-41.

38. Kitamura N, Tohyama $\mathbf{H}$, Nishihara J, et al. Stress deprivation enhances manganese superoxide dismutase expression in the rat patellar tendon. Int J Mol Med 2004;14:537 43.

39. Uchida H, Tohyama H, Nagashima K, et al. Stress deprivation simultaneously induces over-expression of interleukin-1beta, tumor necrosis factor-alpha, and transforming growth factor-beta in fibroblasts and mechanical deterioration of the tissue in the patellar tendon. J Biomech 2005;38:791-8.

40. Abiezzi SS, Gesink DS, Schreck PJ, et al. Increased expression of the beta 1, alpha 5 and alpha $v$ integrin adhesion receptor subunits occurs coincident with remodeling of stress-deprived rabbit anterior cruciate and medial collateral ligaments. J Orthop Res 1995;13:594-601.

41. Katsumi A, Naoe T, Matsushita T, Kaibuchi K, Schwartz MA. Integrin activation and matrix binding mediate cellular responses to mechanical stretch. J Biol Chem 2005;280:16546-9

42. Wren TA, Beaupre GS, Carter DR. Tendon and ligament adaptation to exercise, immobilization, and remobilization. J Rehabil Res Dev 2000;37:217-24. 